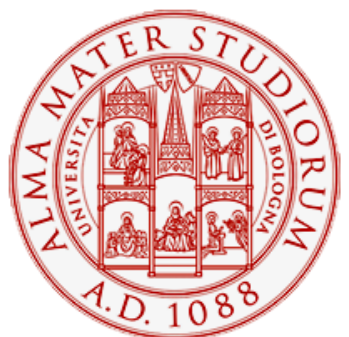

Alma Mater Studiorum - Università di Bologna DEPARTMENT OF ECONOMICS

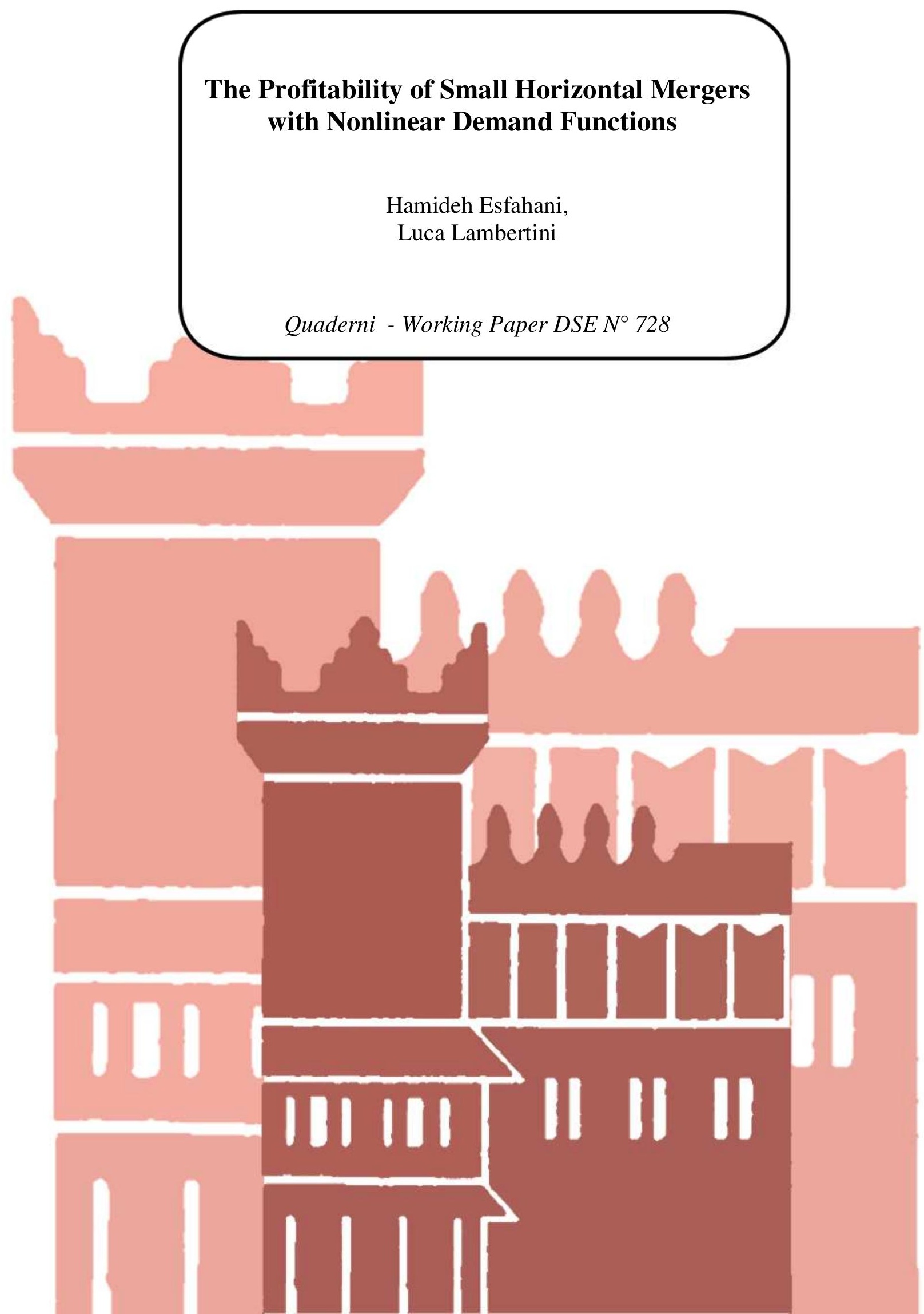




\title{
The Profitability of Small Horizontal Mergers with Nonlinear Demand Functions
}

\author{
Hamideh Esfahani, Luca Lambertini \\ Department of Economics, University of Bologna \\ Strada Maggiore 45, 40125 Bologna, Italy \\ hamideh.esfahani2@unibo.it; luca.lambertini@unibo.it \\ fax +39-051-2092664
}

First draft: June 2010

This draft: January 2011

\begin{abstract}
We want to take a differential game approach with price dynamics to conduct an investigation into the consequences of horizontal merger of firms where the demand function is nonlinear. We take into consideration the open-loop equilibrium. We show that in relation to the fact that the demand is nonlinear and prices follow some stickiness an incentive for small merger exists, while it does not appear under the standard approach using a linear demand function.

Keywords: Horizontal mergers, Differential game, Sticky price, Nonlinear demand

JEL classification: C73, D43, G34, L13
\end{abstract}




\section{Introduction}

The existing literature on the profitability of horizontal mergers within the static Cournot framework demonstrates that a merger will be profitable to the merging firms provided that either the number of merging firms is large enough (typically, too large to be realistic) or the merger creates a strong synergy (typically, a reduction in the average costs bringing about the well known efficiency defense). Convincing illustrations of these claims can be found in Salant, Switzer and Reynolds (1983) and Farrell and Shapiro (1990), respectively. The subsequent contributions have enriched the discussion along much the same lines. Levin (1990) and Cheung (1992), using different setups, demonstrate that at least $50 \%$ of the market should merge to provide a profitable merger. Gaudet and Salant $(1991,1992)$ show that if a subset of firms were to produce below their Cournot equilibrium quantity, their profits would fall if the subset of firms is not large enough. the efficiency argument is first advocated by Perry and Porter (1985), showing that if firms can benefit from some economies of scale the merger will become profitable, and then extensively discussed by Farrell and Shapiro (1990), who find that a horizontal merger that does not generate synergies raises the price and this price increase can make the merger profitable only when the market share of merging firm is large enough.

All of the above is based upon a static approach. There are also studies addressing the profitability of mergers using dynamic models. Dockner and Gaunersdorfer (2001), through a numerical analysis, and Benchekroun (2003), analytically, revisit the differential game introduced by Fershtman and Kamien (1987) to assess horizontal mergers among firms that compete in a dynamic Cournot oligopoly with price adjustments. They consider feedback and open-loop equilibria where the demand function is linear. Cellini and Lambertini (2007) model the optimal capacity and output decisions of oligopolistic firms in a dynamic game with capital accumulation á la Ram-

sey. Borrowing the non-lnear demand structure from Anderson and Engers $(1992,1994)$, they allow for non-linear market demand functions. They use this setup to investigate the role of horizontal mergers in driving the economy towards the Ramsey modified golden rule.

Here, we take an alternative route to the analysis of the incentive to carry out small mergers table when the pre-merging degree of concentration is high but any cost saving through the merger is ruled out by assumption. To do this, we take a differential game approach with sticky price dynamics à la 
Simaan and Takayama (1978) and Fershtman and Kamien (1987), combined with a non-linear demand structure à la Anderson and Engers $(1992,1994)$. Given the shape of the resulting maximization problem, we take into consideration the open-loop equilibrium only, focussing on the interplay between price stickiness and the curvature of demand. The main point we make in this paper boils down to the following: as the market shrinks because demand becomes convex, firms bear increasing profit losses, and this creates an incentive for small mergers that would not appear under the standard approach with a linear demand function. Additionally, our analyis also singles out the existence of a parameter region where the merger between two firms out of three is always profitable, for any degree of price stickiness as well as for some degree of concavity of the demand function.

The remainder of the paper is organized as follows. Section 2 contains the layout of the model. Sections 3 illustrate the pre-merger open-loop equilibrium. The assessment of profit incentives towards small mergers is carried out section 4 . Section 5 concludes.

\section{The setup}

Consider a dynamic oligopoly where $n$ symmetric firms produce the same homogeneous good over continuous time $t \in[0, \infty)$, all of them with the same constant average and marginal $\operatorname{cost} c=0$. The notional market demand function is defined as follows:

$$
Q(t)=A-(\hat{p}(t))^{\alpha} \quad, \alpha>0 .
$$

The above function is always downward sloping, and can be either convex $(0<\alpha<1)$ or concave $(\alpha>1)$. If $\alpha=1$, it is linear. In each period, the market price $\hat{p}(t)$ is determined by the following inverse demand function:

$$
\hat{p}(t)=(A-Q(t))^{\frac{1}{\alpha}} .
$$

where $Q(t)=\sum_{i=1}^{n} q_{i}(t)$ is the industry output and $q_{i}(t) \geq 0, i \in\{1,2, \ldots, n\}$ is the individual output of firm $i$ at time $t$.

However, since price is sticky, the actual market price does not adjust instantaneously to the notional price level (1). That is, $\hat{p}(t)$ will differ from 
the current price level $p(t)$ at any time except that in steady state, with the price moving according to the following equation:

$$
\frac{d p(t)}{d t} \equiv \dot{p}(t)=s\{\hat{p}(t)-p(t)\}
$$

where $s(0<s<\infty)$ is a constant parameter that determines the speed of adjustment. The lower is $s$, the higher is the degree of price stickiness.

The instantaneous profit function of firm $i$ is

$$
\pi_{i}(t)=p(t) q_{i}(t)
$$

Therefore, the objective of firm $i$ is

$$
\max _{q_{i}(t)} J_{i}=\int_{0}^{\infty} e^{-\rho t} p(t) q_{i}(t) d t
$$

subject to (2) and to the initial condition $p(0)=p_{0}$ and the non-negativity condition $p(t) \geq 0$ for all $t \in[0, \infty)$. The factor $e^{-\rho t}$ discounts future gains, and the discount rate $\rho$ is assumed to be constant and equal across firms.

The elasticity of demand function (1) w.r.t. price, $\varepsilon_{Q, P}$, can be written as follows:

$$
\left|\varepsilon_{Q, P}\right|=-\frac{\partial Q(\alpha)}{\partial p(Q(\alpha))} \cdot \frac{p(Q(\alpha))}{Q(\alpha)}=\frac{\alpha p^{\alpha}}{A-p^{\alpha}} .
$$

In view of the fact that the problem at hand is not defined in a linearquadratic form, we solve the differential game using the open-loop information structure.

\section{The pre-merger Cournot equilibrium}

We consider the sum of the consumer surplus and the individual profits of the present firms as the appropriate measure of the social welfare level. Since the marginal cost is zero, the social welfare is the full integral of the curve up to the otimal level of output, $Q^{*}$ :

$$
S W=\int_{0}^{Q^{*}}(A-z)^{\frac{1}{\alpha}} d z=\frac{\alpha}{1+\alpha}\left[A^{\frac{\alpha+1}{\alpha}}-\left(A-Q^{*}\right)^{\frac{\alpha+1}{\alpha}}\right]
$$


Here, since there is no fixed cost, the merger cannot be carried out and justified on the basis of an efficiency argument, and we are not going to dwell upon whether any merger might be socially acceptable. Accordingly, we will focus solely on the firms' incentives.

In solving the quantity-setting game between profit-seeking agents, we shall focus upon a single representative firm, whose Hamiltonian function is:

$$
H_{i}(t)=e^{-\rho t}\left\{q_{i}(t) p(t)+\lambda(t) s\left[\left(A-q_{i}(t)-Q_{-i}(t)\right)^{\frac{1}{\alpha}}-p(t)\right]\right\},
$$

where $\lambda(t)=\mu(t) e^{\rho t}, \mu(t)$ being the co-state variable associated to $p(t)$.

As a first step, we can prove the following:

Proposition 1 At the open-loop Nash equilibrium, the steady state levels of the price and the output of firm $i$ are

$$
\begin{gathered}
q^{*}=\frac{\alpha A(\rho+s)}{s+\alpha n(\rho+s)}, \\
p^{*}=\left(A-n q^{*}\right)^{\frac{1}{\alpha}} .
\end{gathered}
$$

Proof. Taking the first-order condition (FOC) on (5) w.r.t. $q_{i}(t)$, we obtain

$$
p(t)-\frac{1}{\alpha} s \lambda(t)\left(A-q_{i}(t)-Q_{-i}(t)\right)^{\frac{1}{\alpha}-1}=0 .
$$

which seems to be unsolvable because of the exponent $\frac{1}{\alpha}-1$. In general, what is done is to take the FOC, solve it w.r.t. the control variable and then differentiate w.r.t. time. What we actually want is not the explicit solution of (6) w.r.t. $q_{i}(t)$, but rather a control equation describing the evolution of the individual output over time. Hence, we can differentiate the FOC (6) w.r.t. time in the first place and then, by introducing a symmetry condition $q_{i}(t)=q_{j}(t)=q(t)$ for all $i, j$ on outputs, we get the control equation as follows:

$\frac{d q(t)}{d t}=\dot{q}(t)=\frac{(A-n q(t))^{1-\frac{1}{\alpha}}\left[\alpha s \dot{\lambda}(t)(A-n q(t))^{\frac{1}{\alpha}}-\alpha^{2}(A-n q(t)) \dot{p}(t)\right]}{n s(1-\alpha) \lambda(t)}$. 
Then, we can solve the FOC (6) to obtain optimal value of shadow price at any instant $t$ :

$$
\lambda(t)=\frac{\alpha p(t)(A-n q(t))^{1-\frac{1}{\alpha}}}{s} .
$$

Now, we differentiate the Hamiltonian w.r.t. the state variable to build up the co-state equation:

$$
\begin{gathered}
-\frac{\partial H_{i}(t)}{\partial p(t)}=-q(t)+\lambda(t) s=\frac{\partial \mu(t)}{\partial t} \\
\Longrightarrow \dot{\lambda}(t)=\frac{\partial \lambda(t)}{\partial t}=\lambda(t)(s+\rho)-q(t),
\end{gathered}
$$

and finally we have to account for the transversality condition:

$$
\lim _{t \rightarrow \infty} \mu(t) \cdot p(t)=0 \text {. }
$$

Now observe that (2) and (8) together with the (9) can be plugged into (7) to rewrite the control equation explicitly. The stationarity condition at the steady state equilibrium requires (i) $\dot{q}(t)$ to be equal to zero, and (ii) $p(t)$ to be equal to $\hat{p}$, so that the resulting coordinates of the unique open-loop equilibrium point are:

$$
\begin{gathered}
q^{*}=\frac{\alpha A(\rho+s)}{s+\alpha n(\rho+s)}, \\
p^{*}=\left(A-n q^{*}\right)^{\frac{1}{\alpha}} .
\end{gathered}
$$

This concludes the proof.

The resulting per-firm profit in steady state is

$$
\pi^{*}(n)=\frac{\alpha(\rho+s)}{s}\left(\frac{s A}{s+\alpha n(\rho+s)}\right)^{\frac{\alpha+1}{\alpha}}
$$

which is a function of the vertical intercept of demand, time discounting, the number of firms, the degree of price stickiness and the curvature of demand. We are now ready to assess the profitability of a small horizontal merger. 


\section{The incentive to merge}

The puzzle of bilateral mergers in a Cournot tripoly is a recurrent theme in the debate on mergers, as observation suggests that indeed this is precisely the type of merger one happens to observe in reality. For example, this has been the case with the merger between Boeing and McDonnell Douglas that has turned the world industry for large civil air transport into a duopoly, the other competitor being Airbus. To tackle this issue, we set $n=3$ and focus on the profitability of a merger involving two firms out of three. In a triopoly, two firms will find it profitable to merge horizontally if and only if the following condition is satisfied:

$$
\frac{\pi^{*}(n=2)}{2}-\pi^{*}(n=3)>0
$$

or, equivalently,

$$
\frac{1}{2}\left(\frac{s A}{s+\alpha 2(\rho+s)}\right)^{\frac{\alpha+1}{\alpha}}-\left(\frac{s A}{s+\alpha 3(\rho+s)}\right)^{\frac{\alpha+1}{\alpha}}>0 .
$$

That is, the fifty percent of the individual duopoly profits once the merger has taken place must be higher than the individual profits before the merger. Since the level of the reservation price $A$ is irrelevant, the above condition is indeed equivalent to the following:

$$
\frac{1}{2}\left(2 \rho+\left(2+\frac{1}{\alpha}\right) s\right)^{-\frac{\alpha+1}{\alpha}}-\left(3 \rho+\left(3+\frac{1}{\alpha}\right) s\right)^{-\frac{\alpha+1}{\alpha}}>0
$$

The above condition can be studied in the space $(\alpha, s / \rho)$. This is done in Figure 1, where the region in which inequality (10) holds, or in other words the merger of two out of three firms is profitable, is represented by the area below the curve.

This figure shows that if the market is sufficiently small (for all $\alpha<0.42$, which is the asymptotic value attained if $s / \rho$ becomes infinitely high), then the incentive for a horizontal merger involving two firms out of three always exists irrespective of speed of adjustment. The essential reason is that when demand becomes convex, the market shrinks and this clearly hinders firms' profitability, a fact which creates an incentive to merge. Interestingly, this result extends to the static game for the same reason. If the prices adjusts instantaneously, then the limit of the open-loop setup is the static model (the 
same happens if $\rho$ tends to zero). This can be verified simply by taking the static model and compare it with the limit case where the ratio $s / \rho$ tends to infinity (see Cellini and Lambertini, 2004). Figure 1 clearly shows that, in the static game where $s / \rho$ shoots up to infinity, the merger is indeed profitable for all $\alpha \in(0,0.42)$.

Figure 1 : Merger profitability in the space $(\alpha, s / \rho)$

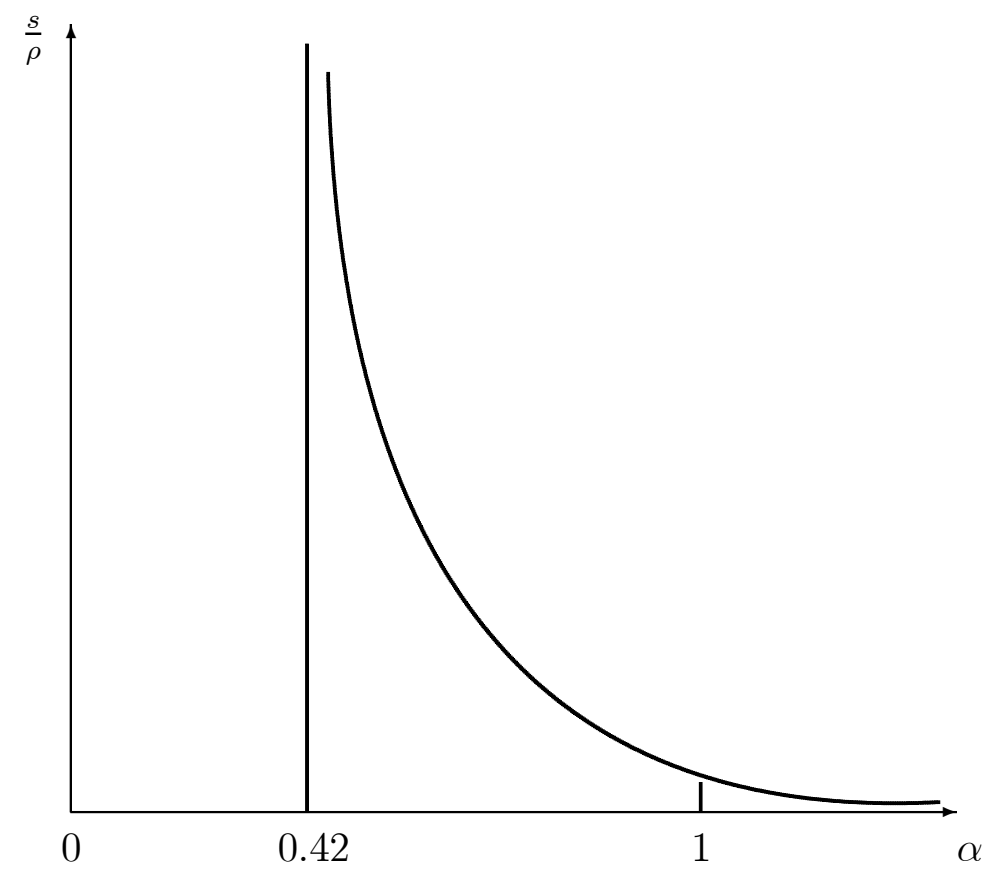

As the market becomes larger (i.e., $\alpha$ increases), the incentive to merge is determined by how fast the price adapts to the equilibrium level. In fact, it turns out that the profitability of the merger is driven by a sort of tradeoff between the curvature of demand and the speed of price adjustment (weighted for the discount rate): as the market enlarges, the price has to become stickier in order for forms to be willing to merge. Put it differently, for comparatively higher values of $\alpha$ one would be tempted to exclude investigating the merger incentive because operating in a larger market increases the standalone profitability and this points in the direction of making bilateral mergers unprofitable, all else equal. This is not the case if the increase in $\alpha$ goes along with a decrease in $s / \rho$. The intuition behind this mechanism can be spelled 
out as follows. When price adjusts very slowly, this involves making large systematic mistakes in setting the output levels. Firms would like to play the correct Cournot equilibrium with a large market but they cannot because $s$ is very small and there they have an incentive to decrease the number of firms to recover what they are losing. This creates a region where $\alpha>1$ and $s / \rho$ is sufficiently small to yield convenient bilateral mergers, as Figure 1 indeed illustrates.

The problem can also be approached from another angle, namely, by fixing $s / \rho$ and then evaluating the profitability of a merger involving $m$ firms out of $n$. In such a case, the profit incentive exists iff:

$$
\frac{\pi^{*}(n-m+1)}{m}-\pi^{*}(n)>0
$$

or, equivalently,

$$
\frac{1}{m}\left(\frac{1}{s+\alpha(n-m+1)(\rho+s)}\right)^{\frac{\alpha+1}{\alpha}}-\left(\frac{1}{s+\alpha n(\rho+s)}\right)^{\frac{\alpha+1}{\alpha}}>0
$$

Condition (11) is assessed in Figure 2, where it yields a parametric family of curves, between the two curves depicted here, with the characteristic that the region where the merger is profitable, which is bellow the curves, enlarges if stickiness and discounting become higher which make sense according to the aforementioned reasons.

This figure says that if $\alpha$ is sharply below 1, a small merger is indeed privately convenient. This makes sense because market size shrinks as $\alpha$ decreases. Now, if the market becomes smaller, because the demand from linear becomes convex, it becomes less profitable. Consequently, it is easier to find conditions whereby a small merger works. Therefore, for a given initial population of firms, say 10, if they are squeezed inside a very small market, then some of them find it profitable to merge because their profits are squeezed by the decrease in $\alpha$, while they would not if the market were larger. In the linear case when $\alpha$ is equal to 1 (along the flat line drawn in Figure 2), we know from Salant, Switzer and Reynolds (1983) that a profitable merger must involve about $80 \%$ of the oligopolist, which is dramatically close to a merger to monopoly. 
Figure 2 : Merger profitability in the space $(m, \alpha)$

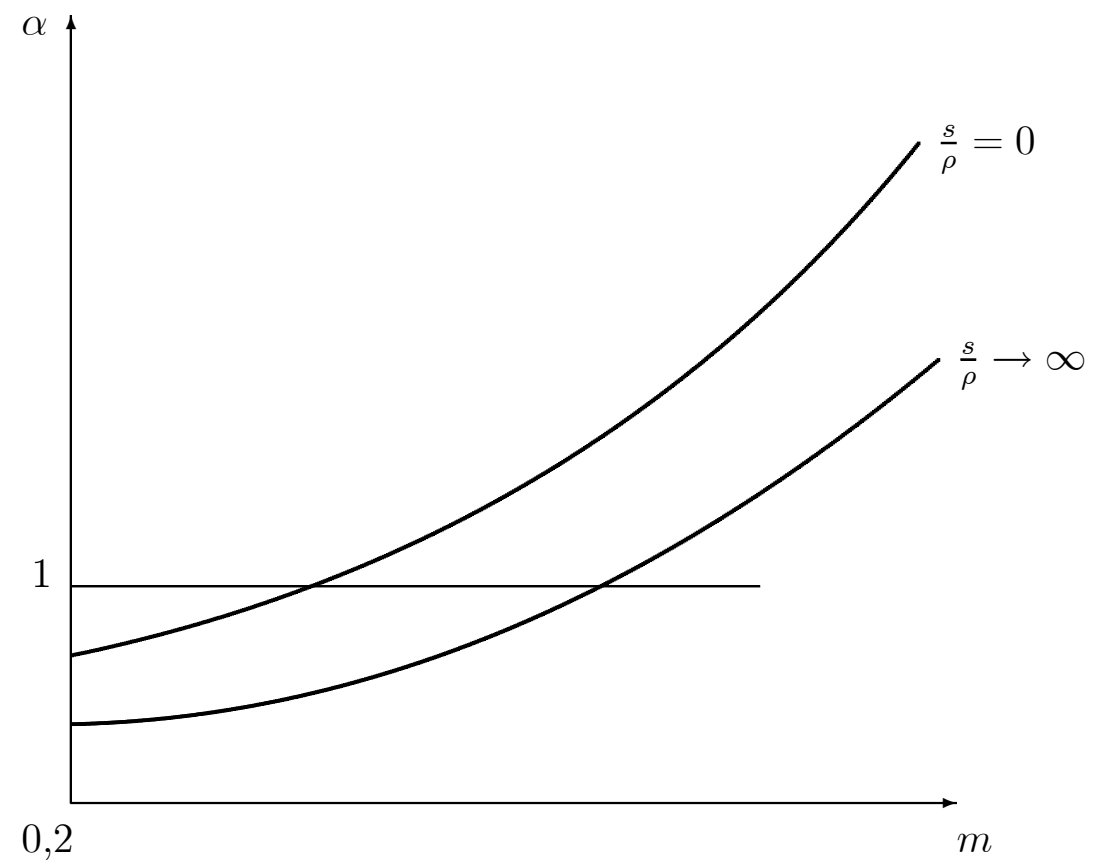

\section{Conclusion}

In this paper, we have investigated the consequences of horizontal mergers taking a differential game approach with sticky prices where the demand function is nonlinear. Given the shape of the problem at hand, we have analyzed the open-loop solution only.

Efficiency implications being ruled out by assumption, in our model it is clear that any decrease in the number of firms brings about a decrease in social welfare via a decrease in consumer surplus. Given the fact that, in principle, when there is no saving on the overall industry costs, any horizontal merger is socially harmful, then the regulator must look out for mergers driven by the pressure generated by market size on firms, when the demand is non-linear. This has some interesting implications on the empirical side, as estimating market demand functions may indeed yield clearcut hints as 
to whether the market under consideration is likely to generate incentives towards horizontal mergers. 


\section{References}

1. Anderson S.P. and Engers M. (1992), Stackelberg vs Cournot oligopoly equilibrium, International Journal of Industrial Organization, 10, 12735 .

2. Anderson S.P. and Engers M. (1994), Strategic investment and timing of entry, International Economic Review, 35, 833-53.

3. Benchekroun, H. (2003), The closed-loop effect and the profitability of horizontal mergers, Canadian Journal of Economics, 36, 546-65.

4. Cellini, R. and Lambertini, L. (2004), Dynamic oligopoly with sticky prices: Closed-loop, feedback and open-loop solutions, Journal of Dynamical and Control Systems, 10, 303-14.

5. Cellini, R.,Lambertini,L. (2007), Capital accumulation, mergers, and the Ramsey golden rule, in Quincampoix, M., Vincent, T., Jørgensen, S. (Eds.), Advances in Dynamic Game Theory and Applications, Annals of the International Society of Dynamic Games, vol. 8. Birkhauser, Boston, 487-505.

6. Cheung, F.K. (1992), Two remarks on the equilibrium analaysis of horizontal mergers, Economics Letters, 40, 119-23.

7. Deneckere, R. and Davidson, C. (1985), Incentives to form coalitions with Bertrand competition, RAND Journal of Economics, 16, 473-86.

8. Dockner, E.J. and Gaunersdorfer, A. (2001), On the profitability of horizontal mergers in industries with dynamic competition, Japan and the World Economy, 13, 195-216.

9. Farrell, J. and Shapiro, C. (1990), Horizontal mergers: An equilibrium analysis, American Economic Review, 80, 107-26.

10. Fershtman, C. and Kamien, M.I. (1987), Dynamic duopolistic competition with sticky prices, Econometrica, 55, 1151-64.

11. Gaudet, G. and Salant, S.W. (1991), Increasing the profits of a subset of firms in oligopoly models with strategic substitutes, American Economic Review, 81, 658-65. 
12. Levin, D. (1990), Horizontal mergers: The 50-percent benchmark, American Economic Review, 80, 1238-45

13. Mehlmann, A. (1988), Applied Differential Games, Plenum Press, New York.

14. Perry, M.K. and Porter, R.H. (1985), Oligopoly and the incentive for horizontal merger, American Economic Review, 75, 219-27.

15. Reinganum, J. (1982), A class of differential games for which the closed loop and open loop Nash equilibria coincide, Journal of Optimization Theory and Applications, 36, 253-62.

16. Salant, S.W., Switzer, S. and Reynolds, R.J. (1983), Losses from horizontal merger: The effects of an exogenous change in industry structure on Cournot-Nash equilibrium, Quarterly Journal of Economics, 98, 185213.

17. Simaan, M. and Takayama, T. (1978), Game theory applied to dynamic duopoly problems with production constraints, Automatica, 14, 161-66. 


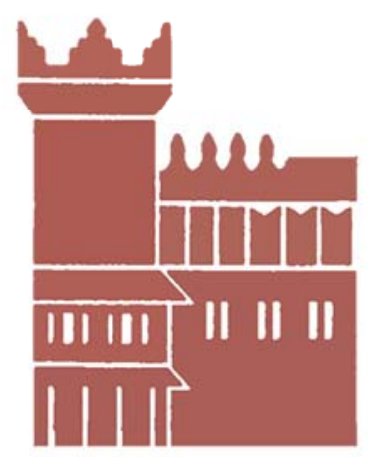

Alma Mater Studiorum - Università di Bologna DEPARTMENT OF ECONOMICS

Strada Maggiore 45

40125 Bologna - Italy

Tel. +39051 2092604

Fax +390512092664

http://www.dse.unibo.it 